TITLE:

\title{
Macroscopic magnetization modeling of silicon steel sheets using an assembly of six-domain particles
}

\section{$\operatorname{AUTHOR}(S)$ :}

Ito, S.; Mifune, T.; Matsuo, T.; Kaido, C.

\section{CITATION:}

Ito, S. ...[et al]. Macroscopic magnetization modeling of silicon steel sheets using an assembly of six-domain particles. Journal of Applied Physics 2015, 117(17): 17 D126.

\section{ISSUE DATE:}

2015-03-20

URL:

http://hdl.handle.net/2433/217018

\section{RIGHT:}

(c) 2015 AIP Publishing. This article may be downloaded for personal use only. Any other use requires prior permission of the author and AIP Publishing. The following article appeared in J.' 'Appl. Phys. 117, $17 \mathrm{D} 126$ (2015)' and may be found at http://scitation.aip.org/content/aip/journal/jap/117/17/10.1063/1.4915105. 


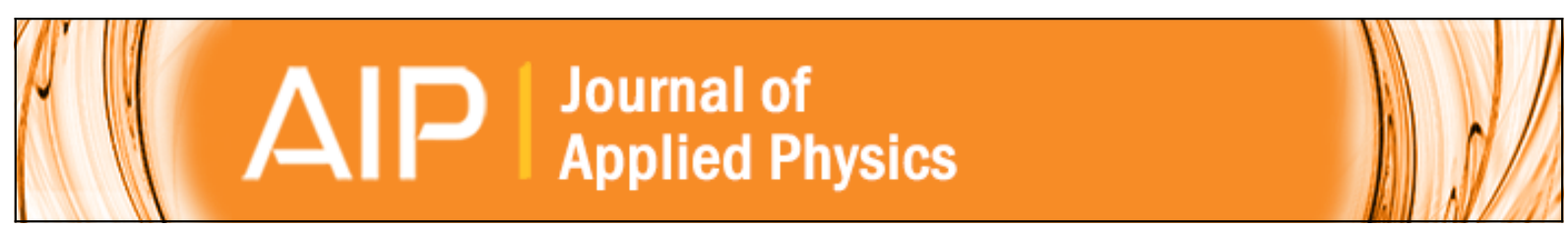

Macroscopic magnetization modeling of silicon steel sheets using an assembly of sixdomain particles

S. Ito, T. Mifune, T. Matsuo, and C. Kaido

Citation: Journal of Applied Physics 117, 17D126 (2015); doi: 10.1063/1.4915105

View online: http://dx.doi.org/10.1063/1.4915105

View Table of Contents: http://scitation.aip.org/content/aip/journal/jap/117/17?ver=pdfcov

Published by the AIP Publishing

\section{Articles you may be interested in}

Magnetization modeling of silicon steel using a simplified domain structure model

J. Appl. Phys. 111, $07 D 107$ (2012); 10.1063/1.3672073

Magnetization process analysis using a simplified domain structure model

J. Appl. Phys. 109, 07D332 (2011); 10.1063/1.3556923

Anisotropic magnetic properties and domain structure in Fe-3\%Si (110) steel sheet

J. Appl. Phys. 109, 07 A307 (2011); 10.1063/1.3535547

Dependence of magnetic susceptibility on stress in textured polycrystalline Fe 81.6 Ga 18.4 and Fe 79.1 Ga 20.9 Galfenol alloys

Appl. Phys. Lett. 96, 012502 (2010); 10.1063/1.3280374

Method to study temperature and stress induced magnetic transitions

Rev. Sci. Instrum. 76, 013910 (2005); 10.1063/1.1832448

Pure Metals • Ceramics Alloys • Polymers in dozens of forms
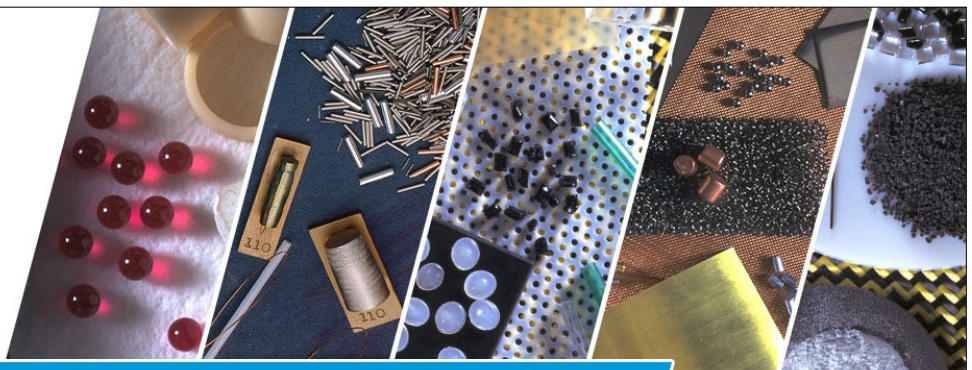


\title{
Macroscopic magnetization modeling of silicon steel sheets using an assembly of six-domain particles
}

\author{
S. Ito, ${ }^{1, a)}$ T. Mifune, ${ }^{1}$ T. Matsuo, ${ }^{1}$ and C. Kaido ${ }^{2}$ \\ ${ }^{1}$ Kyoto University, Kyotodaigaku-katsura, Nishikyo-ku, Kyoto 615-8510, Japan \\ ${ }^{2}$ Kitakyushu National College of Technology, 5-20-1 Shii, Kokuraminamiku, Kitakyushu, \\ Fukuoka 802-0985, Japan
}

(Presented 7 November 2014; received 22 September 2014; accepted 6 November 2014; published online 20 March 2015)

\begin{abstract}
A simplified domain structure model having six domains is proposed for mesoscopic magnetization under cubic anisotropy. The six-domain model represents $90^{\circ}$ and $180^{\circ}$ domain-wall motions by the volume-ratio variations of domains. The magnetization process of grain-oriented and non-oriented silicon steel sheets is represented by the assembly of six-domain models. Simulated magnetization curves agree well with measured properties, and the effect of compressive stress is successfully reconstructed. @ 2015 AIP Publishing LLC. [http://dx.doi.org/10.1063/1.4915105]
\end{abstract}

\section{INTRODUCTION}

Macroscopic magnetic characteristics of core materials result from microscopic/mesoscopic magnetization processes ${ }^{1}$ such as magnetic domain-wall motion and magnetization rotation. To constitute mesoscopic magnetization models, several approaches have been proposed, such as the use of phase theory ${ }^{2}$ and the domain structure model. ${ }^{3-6}$

Multiscale approaches have recently been developed to construct a physical macroscopic model. Hubert and Danie ${ }^{7}$ presented a multiscale model based on polycrystalline magnetomechanical modeling and approximate magnetic energy minimization. Sudo et al. ${ }^{8}$ proposed the assembly of simplified domain structure models (SDSMs). The former model has successfully represented the macroscopic anhysteretic magnetic property of a grain-oriented (GO) silicon steel sheet including the magnetoelastic property. However, its advanced modeling has a large computational cost. The latter model, called the assembled domain structure model (ADSM), gives a physical description of the macroscopic hysteretic magnetization of core materials using a simple assembly of mesoscopic particles. However, it is difficult for the SDSM having two domains to represent the transition between $180^{\circ}$ and $90^{\circ}$ domain-wall motions in the presence of cubic crystalline anisotropy.

This paper proposes the ADSM having six domains to realistically represent a magnetization process, where magnetization vectors in the six domains are directed nearly parallel/antiparallel to the three easy axes. The magnetization process of GO and non-oriented (NO) silicon steel sheets is represented by the proposed model.

\section{SIX-DOMAIN SDSM}

Assuming cubic crystalline anisotropy, this paper examines the SDSM having six domains to represent $90^{\circ}$ and $180^{\circ}$ domain-wall motions by varying the volume ratios of the

\footnotetext{
a) Author to whom correspondence should be addressed. Electronic mail: ito.shumpei.64u@st.kyoto-u.ac.jp.
}

domains (Fig. 1). The six domains correspond to directions parallel/antiparallel to the three easy axes. The magnetization is represented by normalized magnetization vectors $\left(\boldsymbol{m}_{1}, \ldots, \boldsymbol{m}_{6}\right)$ and volume ratios of domains $\left(r_{1}, \ldots, r_{6}\right)$, where $r_{6}=1-r_{1}-r_{2}-r_{3}-r_{4}-r_{5}$. The magnetization vector $\boldsymbol{m}_{i}$ is given by $\boldsymbol{m}_{i}=\left(\sin \theta_{i} \cos \phi_{i}, \sin \theta_{i} \sin \phi_{i}, \cos \theta_{i}\right)$. When a volume ratio $r_{i}$ is 0 , the magnetization vector $\boldsymbol{m}_{i}$ is set to the designated direction along one of the easy axes. The variables $\boldsymbol{X}=\left(\theta_{1}, \ldots, \theta_{6}, \phi_{1}, \ldots, \phi_{6}, r_{1}, \ldots, r_{5}\right)$ are determined by finding a local minimum of total magnetic energy (Fig. 1).

The total energy is normalized by the crystalline anisotropic energy. The normalized total energy $e$ consists of the Zeeman energy, crystalline anisotropic energy, magnetostatic energy, and magnetoelastic energy. The domain-wall energy is neglected in this study by assuming large domain size.

The Zeeman energy is given as

$$
e_{\mathrm{ap}}=-2 \boldsymbol{h} \cdot \sum_{i=1}^{6} r_{i} \boldsymbol{m}_{i},
$$

where $\boldsymbol{h}=\boldsymbol{H}_{\text {ap }} / \kappa M_{\mathrm{S}}$ is the normalized applied field, $\boldsymbol{H}_{\text {ap }}=H_{\text {ap }}\left(\cos \phi_{\text {ap }}, \sin \phi_{\text {ap }}, 0\right)$ is the applied magnetic field, $M_{\mathrm{S}}$ is the magnitude of spontaneous magnetization, $\kappa=2 \mathrm{~K} /$ $\mu_{0} M_{\mathrm{S}}^{2}$, and $K$ is the anisotropy constant.

The normalized crystalline anisotropic energy is given as

$$
e_{\mathrm{an}}=\sum_{i=1}^{6} r_{i} f_{\mathrm{an}}\left(\theta_{i}, \phi_{i}\right)
$$

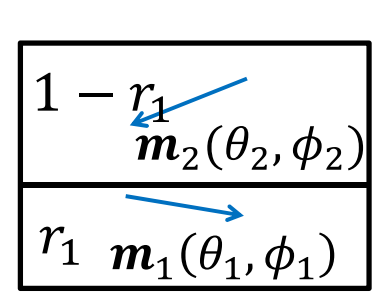

(a)

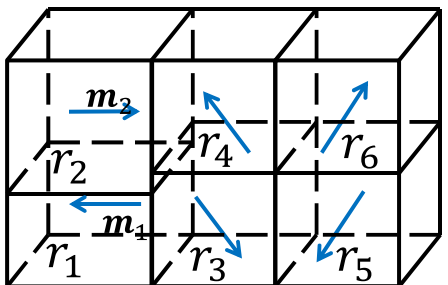

(b)
FIG. 1. Simplified domain structure model: (a) two-domain model and (b) six-domain model. 
where $f_{\text {an }}$ represents the angular dependence. The cubic anisotropy is represented as

$$
f_{\text {an }}\left(\theta_{i}, \phi_{i}\right)=\alpha_{1, i}^{2} \alpha_{2, i}^{2}+\alpha_{2, i}^{2} \alpha_{3, i}^{2}+\alpha_{3, i}^{2} \alpha_{1, i}^{2},
$$

where $\left(\alpha_{1, i}, \alpha_{2, i}, \alpha_{3, i}\right)$ are the direction cosines of the magnetization vectors of the domain $i$ with respect to the three easy axes.

The SDSM assumes that the demagnetization field is uniformly given by the multiplication of demagnetizing factors and the average magnetization. The magnetostatic energy is given as

$$
\begin{gathered}
e_{\mathrm{st}}=s_{x} m_{x}^{2}+s_{y} m_{y}^{2}+s_{z} m_{z}^{2}, \\
\left(m_{x}, m_{y}, m_{z}\right)=\boldsymbol{m}=\sum_{i=1}^{6} r_{i} \boldsymbol{m}_{i},
\end{gathered}
$$

where $s_{x}=k_{x} / \kappa, s_{y}=k_{y} / \kappa, s_{z}=k_{z} / \kappa$, and $k_{x}, k_{y}, k_{z}$ are the demagnetizing factors.

The magnetoelastic energy of the domain $i$ is given as

$$
\begin{aligned}
e_{\mathrm{el}, i}= & -\frac{3}{2} \Lambda_{100} r_{i} \sigma\left(\alpha_{1, i}^{2} \gamma_{1}^{2}+\alpha_{2, i}^{2} \gamma_{2}^{2}+\alpha_{3, i}^{2} \gamma_{3}^{2}-\frac{1}{3}\right) \\
& -3 \Lambda_{111} r_{i} \sigma\left(\alpha_{1, i} \alpha_{2, i} \gamma_{1} \gamma_{2}+\alpha_{2, i} \alpha_{3, i} \gamma_{2} \gamma_{3}+\alpha_{3, i} \alpha_{1, i} \gamma_{3} \gamma_{1}\right),
\end{aligned}
$$

where $\sigma$ is the stress, $\Lambda_{100}=\lambda_{100} / K, \Lambda_{111}=\lambda_{111} / K, \lambda_{100}$ and $\lambda_{111}$ are the magnetostriction constants, and $\left(\gamma_{1}, \gamma_{2}, \gamma_{3}\right)$ are the direction cosines of the stress $\sigma$ with respect to the three easy axes. The normalized magnetoelastic energy is given as

$$
e_{\mathrm{el}}=\sum_{i}^{6} e_{\mathrm{el}, i}
$$

The variable vector $\boldsymbol{X}$ is determined by finding a local minimum of total magnetic energy that satisfies $\partial e / \partial \boldsymbol{X}=0$. Sudo et $a l^{8}{ }^{8}$ obtained a local minimum by finding an equilibrium point of the artificial state equation given as

$$
\mathrm{d} \boldsymbol{X} / \mathrm{d} t=\boldsymbol{Y}, \quad \mathrm{d} \boldsymbol{Y} / \mathrm{d} t=-\partial e / \partial \boldsymbol{X}-\beta \boldsymbol{Y},
$$

where $\boldsymbol{Y}$ is an intermediate variable vector and $\beta$ is a dissipation coefficient. An equilibrium point is obtained by the numerical integration of (8), until reaching the steady state.

Figure 2 shows simulated magnetization curves along the [100], [110], and [111] directions. In this simulation, the magnetostatic and magnetoelastic energies are neglected. This result agrees with the theoretical magnetization curves of a single crystal.

Generally, soft magnetic materials have pinning-type hysteresis due to pinning sites such as crystal defects and crystal grain boundaries. When the distribution of pinning sites is assumed uniform, the pinning effect can be represented by the uniform pinning field given as

$$
\begin{aligned}
h_{\mathrm{pnx}} & =\frac{p_{x}}{\eta} S_{\eta x}(\boldsymbol{m}), \quad h_{\mathrm{pny}}=\frac{p_{y}}{\eta} S_{\eta y}(\boldsymbol{m}), \\
h_{\mathrm{pnz}} & =\frac{p_{z}}{\eta} S_{\eta z}(\boldsymbol{m}), \\
\boldsymbol{S}_{\eta}(\boldsymbol{m}) & =\left(S_{\eta x}, S_{\eta y}, S_{\eta z}\right)=\frac{\eta\left(\boldsymbol{m}-\boldsymbol{m}^{0}+\boldsymbol{S}_{\eta}^{0}\right)}{\left.\max \left(\eta, \mid \boldsymbol{m}-\boldsymbol{m}^{0}+\boldsymbol{S}_{\eta}^{0}\right) \mid\right)},
\end{aligned}
$$

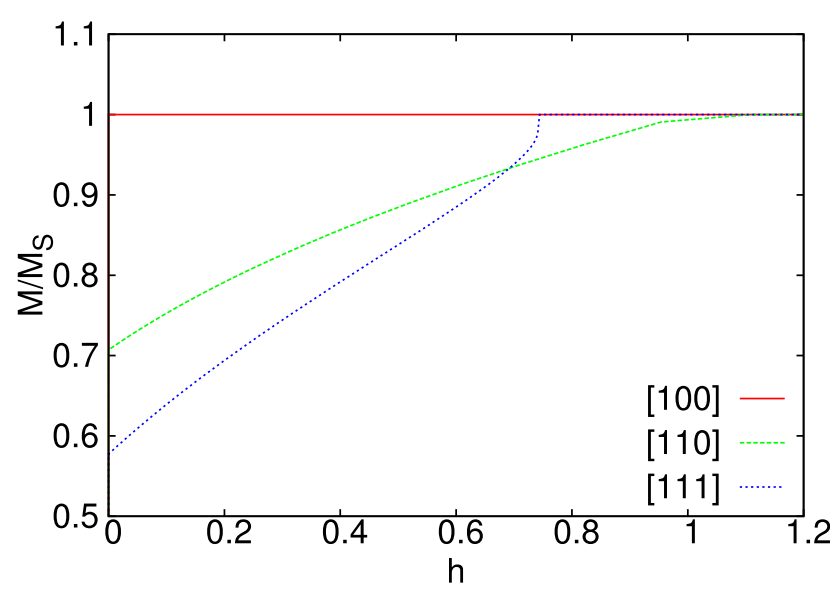

FIG. 2. Simulated magnetization curves along the [100], [110], and [111] directions.

where $\boldsymbol{S}_{\eta}$ is the vector stop hysteron ${ }^{9}$ with radius $\eta,\left(\boldsymbol{m}^{0}, \boldsymbol{S}_{\eta}^{0}\right)$ are the values of $\left(\boldsymbol{m}, \boldsymbol{S}_{\eta}\right)$ at the previous time point, and $p_{x}$, $p_{y}$, and $p_{z}$ are constants that give the magnitude of the pinning field. Taking account of the pinning field similarly to the demagnetization field, pinning-type hysteresis is exhibited by solving $\partial e / \partial \boldsymbol{X}=0$.

Figure 3 shows simulated magnetization curves of a single crystal with $K=3.8 \times 10^{4} \mathrm{Jm}^{-3}, \lambda_{100}=2.3 \times 10^{-5}$, $\lambda_{111}=0, \eta=0.01, \mu_{0} \mathrm{M}_{\mathrm{S}}=2.2 \mathrm{~T}, p_{x}=2.0 \times 10^{-3}, p_{y}=5.0$ $\times 10^{-3}, p_{z}=0$, and $\left(s_{x}, s_{y}, s_{z}\right)=(0.001,0.001,50)$. The three axes are set along the $\boldsymbol{e}_{1}=(1,0,0), \boldsymbol{e}_{2}=(0,1 / \sqrt{2}, 1 / \sqrt{2})$, and $\boldsymbol{e}_{3}=(0,-1 / \sqrt{2}, 1 / \sqrt{2})$ directions as shown in Fig. 4 similarly to those for the GO silicon steel sheet. Tensile stress of $8 \mathrm{MPa}$ due to insulation coating is assumed along the rolling direction (RD).

Simulated magnetization curves along the RD and transverse direction (TD) roughly agree with the property of general GO silicon steel (Fig. 5). Figures 6 and 7 show the simulated magnetization process. Magnetization along the $\mathrm{RD}$ and TD is proceeded mainly by $180^{\circ}$ and $90^{\circ}$ domainwall motions, respectively.

\section{ASSEMBLY OF SDSMs}

To describe the macroscopic magnetization property, an assembly of six-domain SDSMs is examined. Figure 8 shows

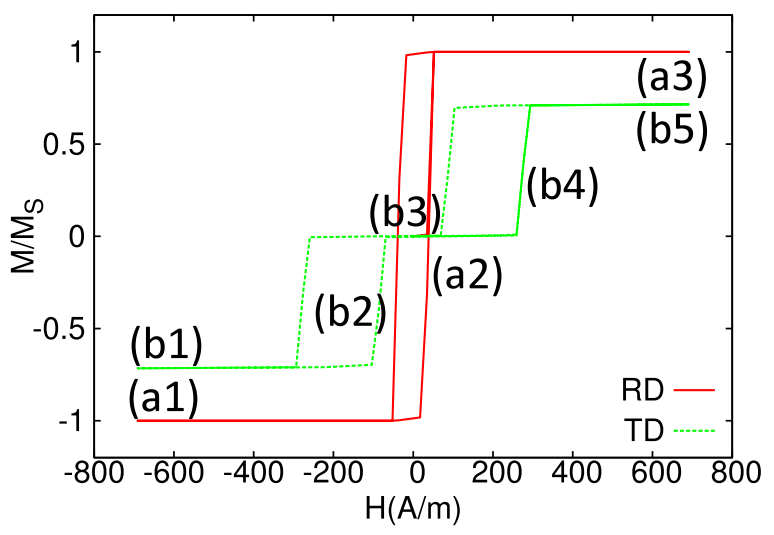

FIG. 3. Simulated magnetization curves of a single crystal along [100] (red line) and $[01 \overline{1}]$ (green line). 


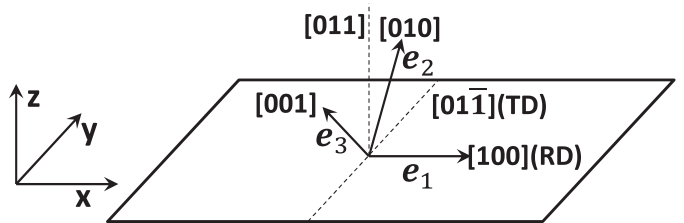

FIG. 4. The three easy axes of the grain-oriented silicon steel sheet.

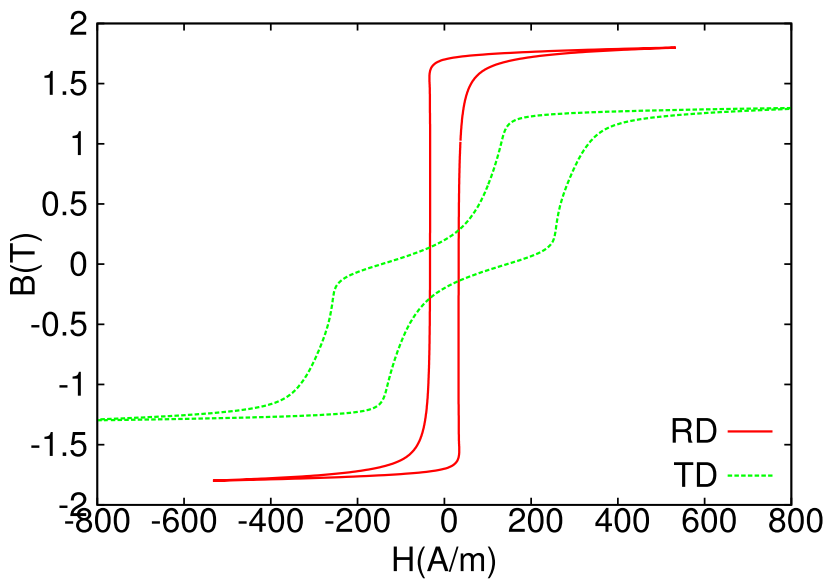

FIG. 5. Measured magnetization curves of the grain-oriented silicon steel sheet.

(a1)

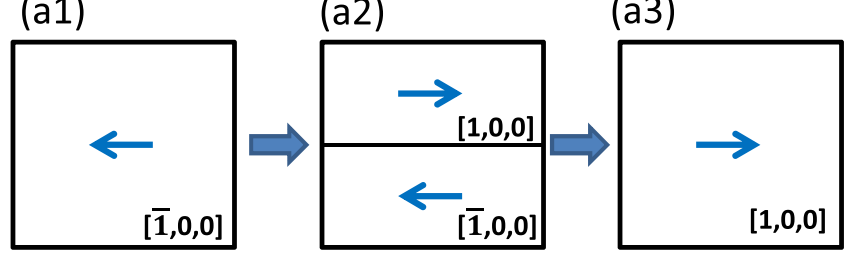

FIG. 6. Magnetization process along the rolling direction.

(b1)

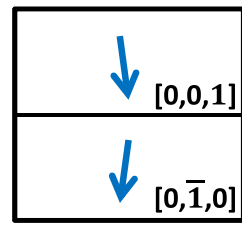

(b5)

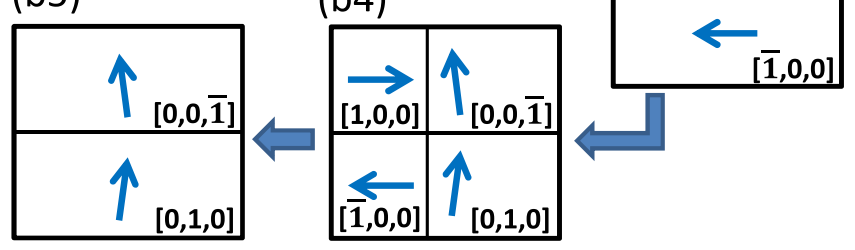

FIG. 7. Magnetization process along the transverse direction.

the ADSM, where each SDSM is regarded as a cell of the model. The Zeeman energy, crystalline anisotropic energy, and magnetoelastic energy are given by the summations of the local energy in each cell.

In the same way as in the micromagnetic simulation, the magnetostatic energy is given by ${ }^{8}$

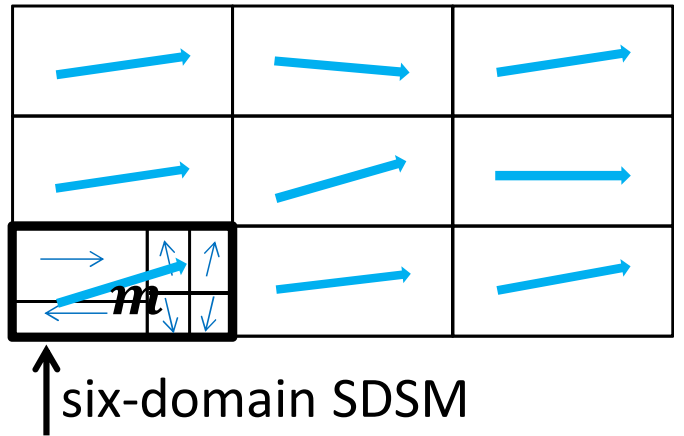

FIG. 8. An assembly of six-domain simplified domain structure models.

$$
e_{\mathrm{st}-\mathrm{global}}=-\sum_{i} \boldsymbol{h}_{\mathrm{st}}(i) \cdot \boldsymbol{m}(i)
$$

where $i$ is the cell index and $\boldsymbol{h}_{\mathrm{st}}$ is the normalized demagnetizing field; $\boldsymbol{h}_{\mathrm{st}}$ is given as

$$
\begin{gathered}
\boldsymbol{h}_{\mathrm{st}}(i)=\sum_{i^{\prime}} \boldsymbol{s}\left(i-i^{\prime}\right) \boldsymbol{m}\left(i^{\prime}\right), \\
\boldsymbol{s}(i)=(1 / \kappa) \boldsymbol{N}(i),
\end{gathered}
$$

where $\boldsymbol{N}(i)$ is the demagnetizing coefficient matrix, which is determined by the geometry of the cell assembly.

\section{SIMULATION RESULT OF THE ADSM}

The magnetization process of the GO silicon steel sheet is simulated using the ADSM with six domains. Tensile stress of $8 \mathrm{MPa}$ along the RD is assumed. The three easy axes are approximately set along the $(1,0,0),(0,1 / \sqrt{2}, 1 / \sqrt{2})$, and $(0,-1 / \sqrt{2}, 1 / \sqrt{2})$ directions, where the axes are distributed within $\pm 5^{\circ}$ randomly. Figure 9 portrays simulated magnetization curves. The material having the dimension ratio of 8 : $8: 10^{-4}$ is divided into $8 \times 8 \times 1$ cells. The magnetization curves are smoother than those given by the single SDSM because of the distribution of crystal orientation and the local demagnetizing field. The difference between the simulated and measured TD magnetization curves is mainly caused by the simple assumptions of uniform pinning field and random distribution of crystal orientation within a limited angle range, which are not very realistic.

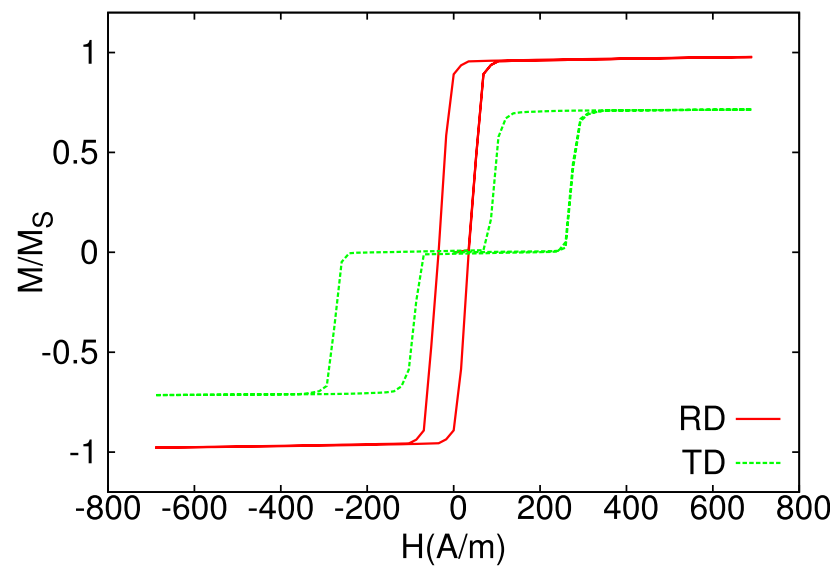

FIG. 9. Simulated magnetization curves of the grain-oriented silicon steel sheet. 


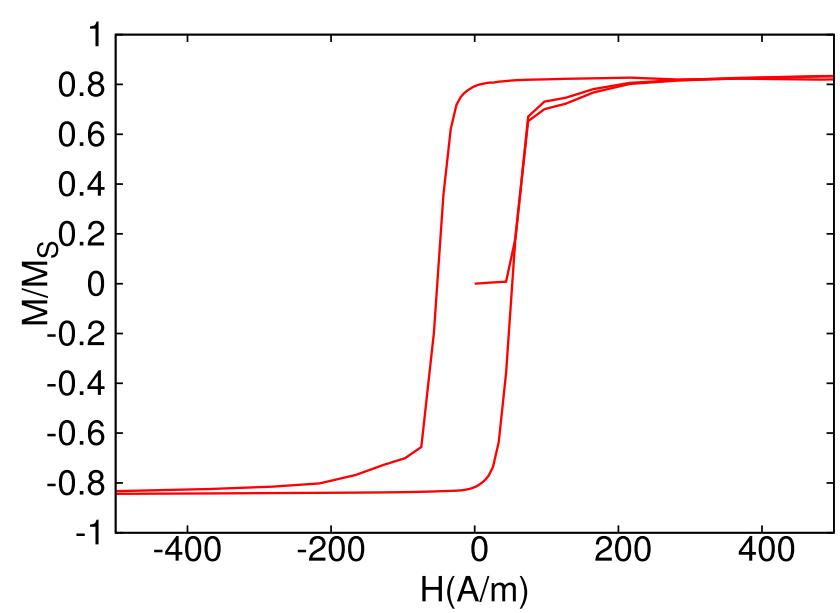

FIG. 10. Simulated magnetization curve of the non-oriented silicon steel sheet.

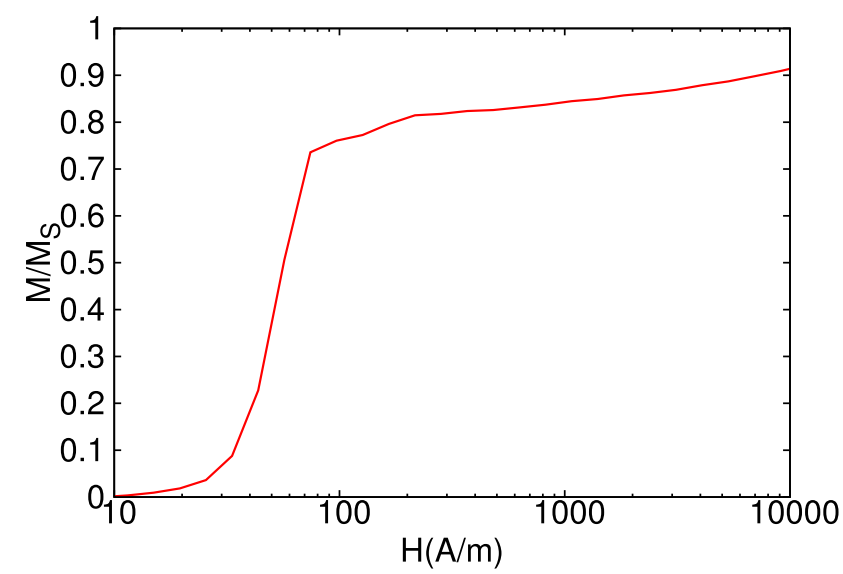

FIG. 11. Simulated anhysteretic curve of the non-oriented silicon steel sheet.

Next, the magnetization process of the NO silicon steel sheet is simulated. The crystal orientation is randomly distributed where one of the easy axes is located in the $x y$-plane. Figures 10 and 11 portray simulated magnetization curves, with $p_{x}=p_{y}=3.0 \times 10^{-3}, p_{z}=0$, and $\eta=0.01$. In Fig. 11, the average magnetization curve $\left(M_{\mathrm{as}}(H)+M_{\mathrm{de}}(H)\right) / 2$ is plotted, where $M_{\mathrm{as}}(H)$ and $M_{\mathrm{de}}(H)$ are the ascending and descending magnetization curves, respectively. Cells having an easy axis close to the RD are magnetized mainly by the $180^{\circ}$ domain wall motion, whereas other cells are magnetized by both $90^{\circ}$ and $180^{\circ}$ domain wall motions. When the applied field is strong, the magnetization rotation becomes dominant.

Figure 12 shows the simulated magnetization curves of NO steel under compressive stress of $0,20,50$, and $100 \mathrm{MPa}$ along the $\mathrm{RD}$, where the average magnetization curves is plotted. Comparison with the measured property shown in Fig. 13 reveals that the reduction in magnetization due to stress is successfully reconstructed.

\section{CONCLUSION}

An SDSM having six domains was proposed. The magnetic properties of GO and $\mathrm{NO}$ silicon steel sheets were successfully represented by the assembly of SDSMs having

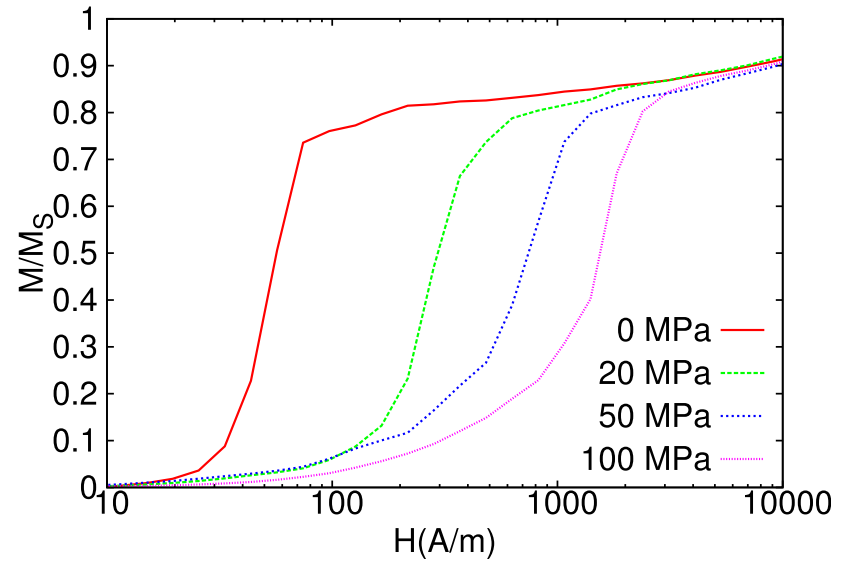

FIG. 12. Simulated magnetization under compressive stress of $\sigma=$ $0,20,50,100 \mathrm{MPa}$.

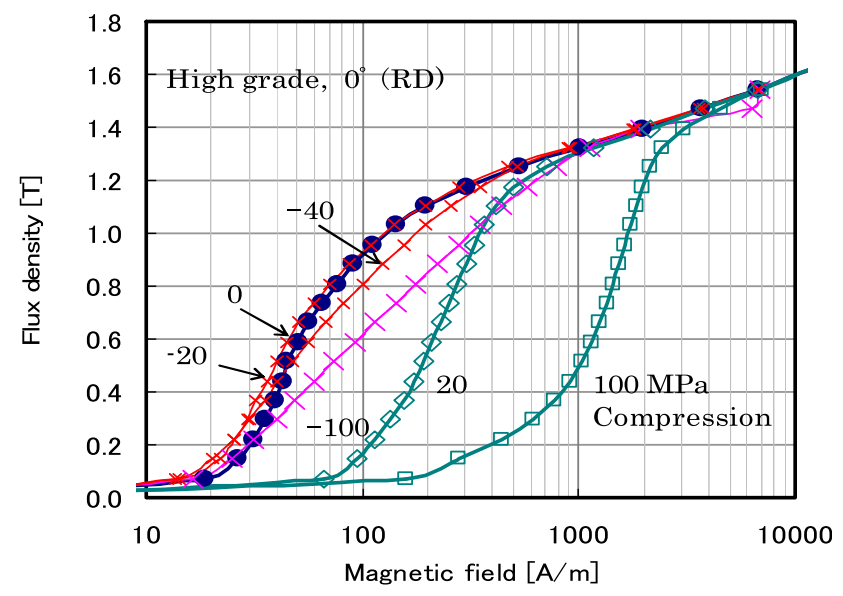

FIG. 13. Measured effects of stress on magnetization curves. ${ }^{10}$

six-domains, where the property degradation due to compressive stress was reconstructed.

\section{ACKNOWLEDGMENTS}

This work was supported in part by the Japan Society for the Promotion of Science under a Grant-in-Aid for Scientific Research (C), Grant No. 26420232.

${ }^{1}$ A. Hubert and R. Schäfer, Magnetic Domains: The Analysis of Magnetic Microstructures (Springer-Verlag, Berlin, Heidelberg, 1998).

${ }^{2}$ G. Bertotti, Hysteresis in Magnetism (Academic Press, 1998).

${ }^{3} \mathrm{~N}$. Smith, "Domain theory model for magnetic thin films," IEEE Trans. Magn. 24, 2380 (1988).

${ }^{4}$ C. Saka, K. Shiiki, and K. Shinagawa, "Simulation of domain structure for magnetic thin film in an applied field," J. Appl. Phys. 68, 263 (1990).

${ }^{5}$ T. Matsuo, "Magnetization process analysis using a simplified domain structure model," J. Appl. Phys. 109, 07D332 (2011).

${ }^{6} \mathrm{M}$. Sudo and T. Matsuo, "Magnetization modeling of silicon steel using a simplified domain structure model," J. Appl. Phys. 111, 07D107 (2012).

${ }^{7}$ O. Hubert and L. Danie, "Multiscale modeling of the magneto-mechanical behavior of grain-oriented silicon steels," J. Magn. Magn. Mater. 320, $1412(2008)$.

${ }^{8}$ M. Sudo, T. Mifune, T. Matsuo, and C. Kaido, “A simplified domain structure model exhibiting pinning field,” IEEE Trans. Magn. 49, 1829 (2013).

${ }^{9}$ T. Matsuo, "Rotational saturation properties of isotropic vector hysteresis models using vectorized stop and play hysterons," IEEE Trans. Magn. 44, 3185 (2008).

${ }^{10}$ C. Kaido, N. Hirose, S. Iwasa, T. Hayashi, and Y. Waki, "Stress dependence of magnetic properties in non-oriented electrical steel sheets," J. Magn. Soc. Jpn. 34, 140 (2010). 